\title{
Coupling Parametric Design and Robotic Assembly Simulation to Generate Thermally Responsive Brick Walls
}

\author{
Sherif Abdelmohsen ${ }^{1,2}$, Khaled Tarabieh ${ }^{1}$, Rana El-Dabaa ${ }^{3}$, Asmaa Hassan ${ }^{4}$, Islam Salem ${ }^{3}$, Yomna \\ El-Ghazi ${ }^{3}$ \\ ${ }^{1}$ American University in Cairo, Cairo, Egypt \\ ${ }^{2}$ Ain Shams University, Cairo, Egypt \\ ${ }^{3}$ Arab Academy for Science, Technology and Maritime Transport, Cairo, Egypt \\ ${ }^{4}$ Future University in Egypt, Cairo, Egypt
}

\begin{abstract}
Recent studies involving advanced custom brickwork configurations using parametric and performance-based approaches in hot arid climates have demonstrated the effect of thermal mass on energy performance, with limited evidence of factors like bonds, patterns and extrusions. This paper addresses generating low-cost thermally responsive masonry walls based on solar radiation data by coupling between thermal simulation using parametric design and robotic assembly simulation. Grasshopper script was used to automatically translate wall configurations resulting from DIVA/ArchSim simulations into robotic assembly simulation using KUKA parametric robot control (PRC). This coupling approach offers unlimited wall texture variation, context responsiveness, and wall construction efficiency and precision as relates to thermal performance.
\end{abstract}

\section{Introduction}

Recent efforts to address advanced brickwork design include parametric approaches (Tarabieh et al., 2017, 2018; Sharif et al., 2015; Cavieres et al., 2016) and performance-based approaches (Fathy et al., 2015; Abdelwahab \& Elghazi, 2016). Efforts in the field of robotics has introduced potentials of application regarding brickwork configuration and assembly (Gramazio et al., 2014; Sousa et al., 2015; Dörfler et al., 2016; Dakhli and Lafhaj, 2017; Usmanov et al., 2017). The added value in such applications relates to high precision fabrication of large-scale walls beyond structured factory settings and the dramatic expansion of application space for automated building construction. These efforts focus on high precision in assembling building components in space, with advanced semi-autonomous fabrication, alignment with existing in-situ components, and sensitivity to dimensional tolerances and site uncertainties. Although relevant to this study, little has been published to explore potentials of coupling between solar radiation maps that directly affect thermal transmittance simulation, specifically in the design, fabrication and assembly of unconventional brickwork configurations.

This paper is part of an ongoing research that aims at investigating, generating and digitally fabricating parametric brick walls that could enhance thermal and energy performance in hot arid climates. However, this paper focuses on the generation of thermally responsive masonry walls based on informed solar radiation data addressing the relation between incident thermal radiation and brick texture (Figure 1). Thermal responsiveness of the wall is related to the variation in thermal mass of wall through differences in bricks extrusion values in response to the shade and shadow from the surrounding context and bricks topography. Moreover, this study presents a coupling approach between parametric tools and robotic assembly simulation to deliver the thermally responsive brick walls.

In previous work, authors analyse the impact of different types of brick bonds on energy consumption in space. English, Flemish and Running bonds were compared in a hot arid climate and concluded that Flemish bond with either $30 \%$ wall area extruded with half brick length, or $60 \%$ bricks extruded with quarter brick length decrease energy consumption with $26 \%$ than other types of bonds. The team explored the impact of brick bonds and integration of parametric analysis with energy performance simulations on a south façade for a typical residential room in a hot arid climate (Tarabieh et al., 2018).

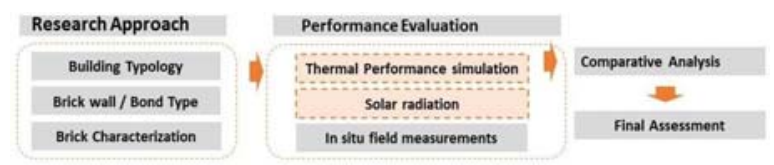

Figure 1: Research Approach.

Initial simulation phases developed a performancedriven brick façade design with an optimal case at 94.6 $\mathrm{kWh} / \mathrm{m}^{2}$ energy consumption (Figure 2) (Tarabieh et al., 2018). A script was developed to translate the relation between incident thermal radiation and brick topography in the optimal masonry wall configuration (at brick extrusion $60 \%$ with non-uniform gradient distribution based on an attractor curve logic) and the required coupling for the simulation of the robotic assembly. Results were divided into two types; either extruded bricks along edges or corners of the facade as shown in Figure 2.

The approach in this paper contributes to the development of façade composition, offering (1) unlimited variation in brick wall texture, (2) responsiveness to surrounding context, (3) efficiency of wall construction in relation to thermal performance through studying solar radiation map by utilizing two simulation parameters self-shading and thermal mass of bricks extrusion value and position, and (4) generation of unique architectural vocabulary by creating custom solar radiation wall topography. 


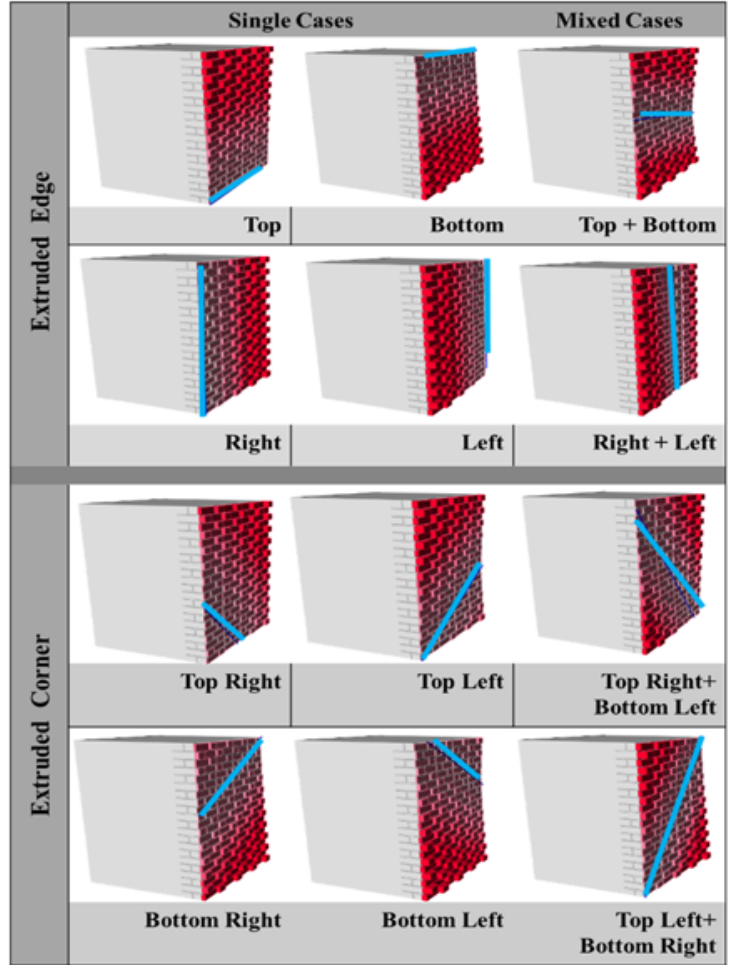

Figure 2: Brick extrusion configurations between extruded bricks from edges or corners (Tarabieh et al., 2018).

\section{Methodology}

The methodology implemented here is divided into three main sections; Thermal simulation using parametric design, Robotic assembly simulation and Solar radiation simulation.

\section{Thermal Simulation using Parametric Design}

This section focuses on integrating solar radiation analysis with thermal simulation using parametric tools to investigate thermal performance of different brick textures (Figure 3).

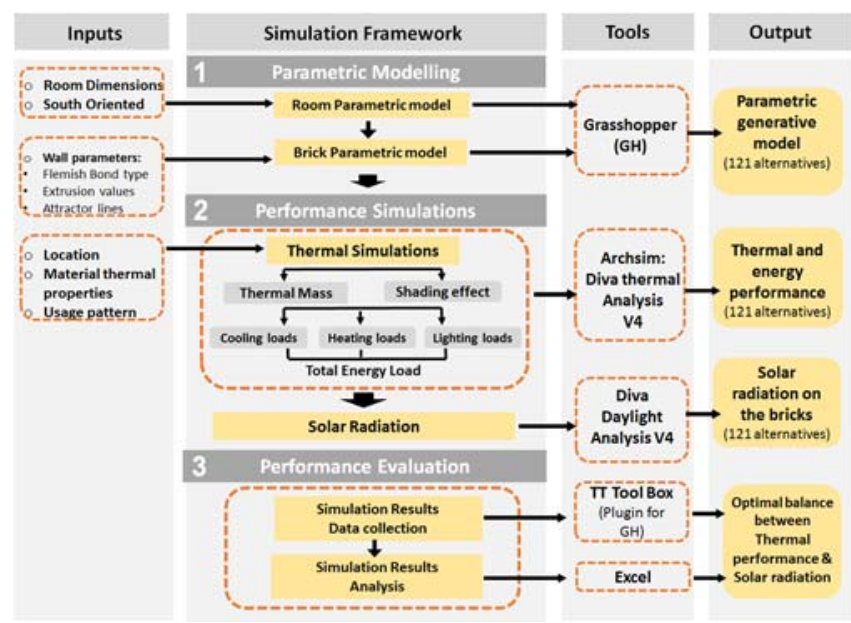

Figure 3: Research Methodology.

Several configurations were studied for brick extrusion through attractor curve parametric logic. The attractor line resembled an axis through the wall that affects the extrusion values according to the distance between each brick midpoint and its closest point on line. The attractor curve has the flat bricks with zero extrusion values, with gradient stepped extrusion values with $1 \mathrm{~cm}$ increments with maximum $10 \mathrm{~cm}$ extrusion in the header brick and $0.5 \mathrm{~cm}$ increments in the stretcher bricks with maximum value of $5 \mathrm{~cm}$. The maximum values of extrusion are related to $1 / 2$ brick extrusion value that varies according to its orientation in the Flemish bond either header or stretcher, for extruded bricks structure stability. A parametric script was used for locating the attractor curve to pass through all possible variations of the wall. The outer rectangle of wall was split into two L-shaped curves with two movable points that construct the location of curve. Each outer edge of the rectangle has five points, each two opposite points create one variation for the location of attractor curve. Different location configurations were tested with 121 cases (Figure 4).
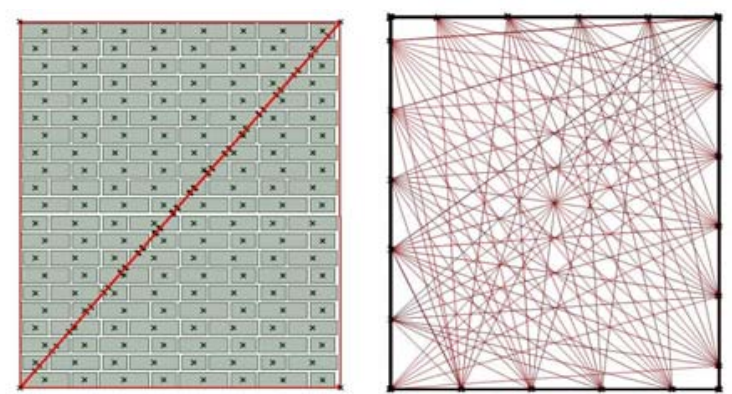

Figure 4: Left: Logic of attractor line cases, Right: All tested cases of attractor curve location (Tarabieh et al., 2018).

In the first section, three stages were addressed for different brick texture that were graded ascending from nearly-smooth brick wall texture to more rough brick wall texture, demonstrating the relation between brick texture, solar radiation exposure and thermal performance. The case study was chosen in Cairo, Egypt $\left(30^{\circ} 6^{\prime} \mathrm{N}, 31^{\circ} 24^{\prime} \mathrm{E}\right.$, alt. $\left.75 \mathrm{~m}\right)$, which belongs to a subtropical desert arid hot climate characterized by high direct solar radiation and clear sky that demands special façade treatments to minimize solar heat gain. The diversity of parameters controlling the gradientbased patterns required the brick facade to be constructed in Grasshopper to control each variable parametrically. DIVA-for-Rhino, a highly optimized daylight and energy simulation tool that interfaces with Radiance/Daysim for daylight and EnergyPlus for thermal simulations (McNeel 2010; Jakubiec and Reinhart, 2011) were utilized to conduct the simulation runs. Simulation results were analysed to evaluate the solar radiation exposure behaviour of the brick configurations and their relationship with both brick wall texture and thermal performance. The simulation parameters are shown in Table 1. To simulate the proposed cases, ArchSim plugin within DIVA-for-GH (Dogan, 2013), was used to interface with EnergyPlus for thermal simulation. 
Table 1: Simulation parameters.

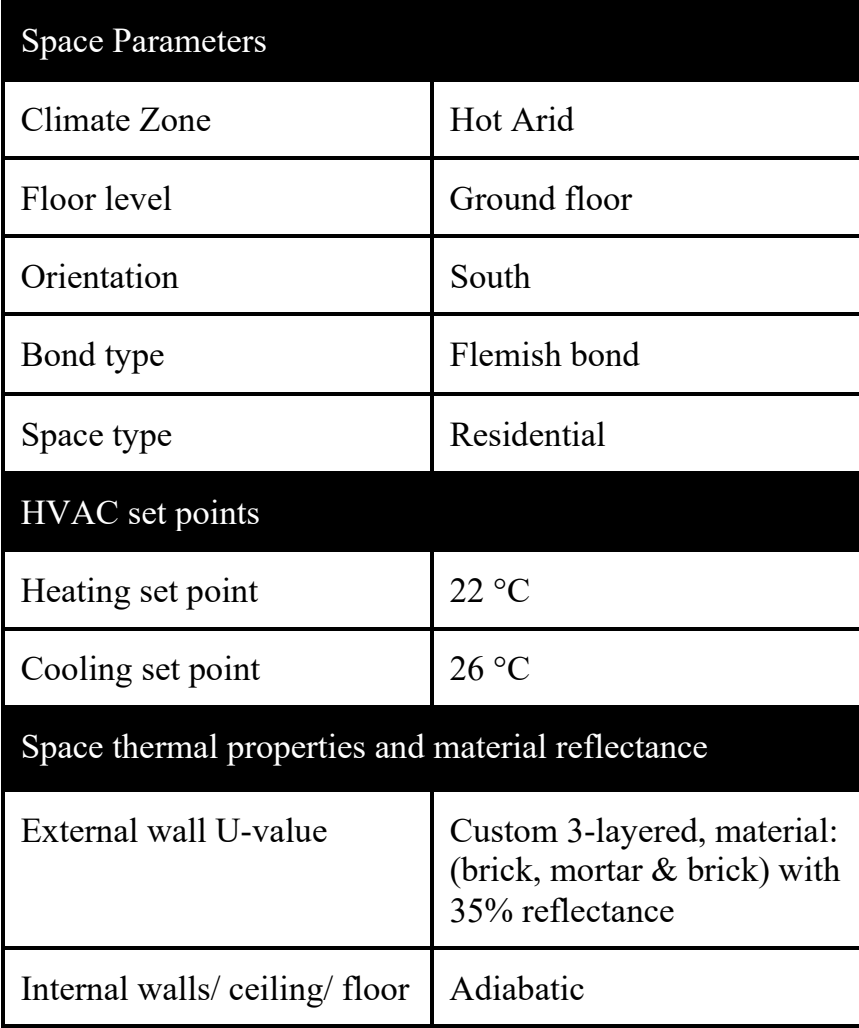

All details were modelled for simulation (Figure 5). The model was divided into two parts; fixed (room, inner bricks layer and mortar layers), and parametric (outer brick layer, and header and stretcher layers), that interact to the attractor line shown in Figure 3. Special algorithms were needed to ensure both the thermal mass effect of the parametric extrusions of the different cases as well as the shading effect of each brick to the other nearby bricks.

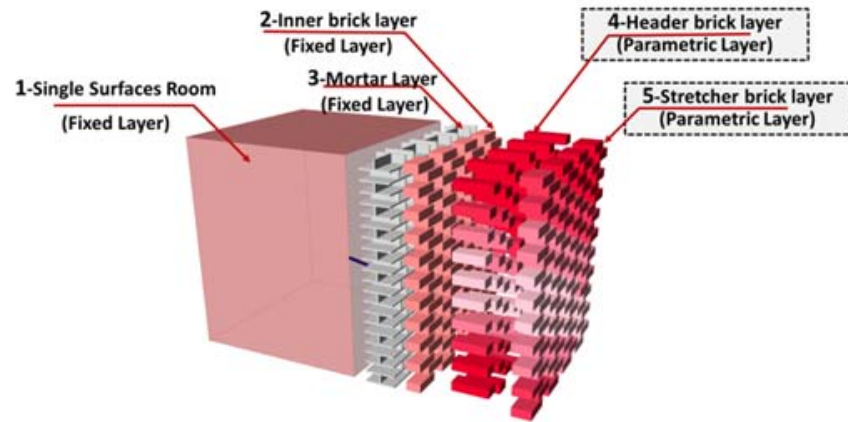

Figure 5: Detailed 3D model of fixed \& parametric layers.

Thus, first, all the fixed layers were embedded in the single surfaces model as an input material. The outer facade material consisted of three layers; the inner bricks layers of $10 \mathrm{~cm}$, the middle mortar layer of $1 \mathrm{~cm}$, and the outer brick layer of different thickness depending on the parametric brick configuration (Figure 6.B: No. 3, 2 and 1 respectively). A special algorithm was conducted to calculate the total thermal mass of all the different extruded bricks to be connected to the above-mentioned parametric material layer (Figure 6.A).

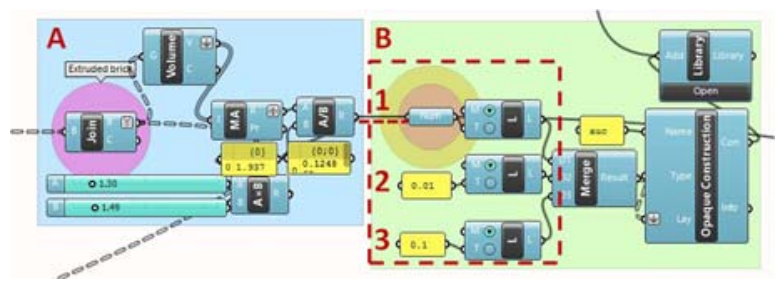

Figure 6: Left (A): Algorithm for calculating thermal mass of total extruded bricks, Right (B): 3 façade layers showing fixed thickness layers (No. 2 \& 3) and parametric layer (No. 1), connected to algorithm.

Second, to ensure the shading effect of each brick to nearby bricks, the 3D model of parametric brick cases of the different attractor lines was modeled and connected to the thermal model using "Shading geometry collection" input within the "Zone Connectivity Network" battery of ArchSim (Figure 7).

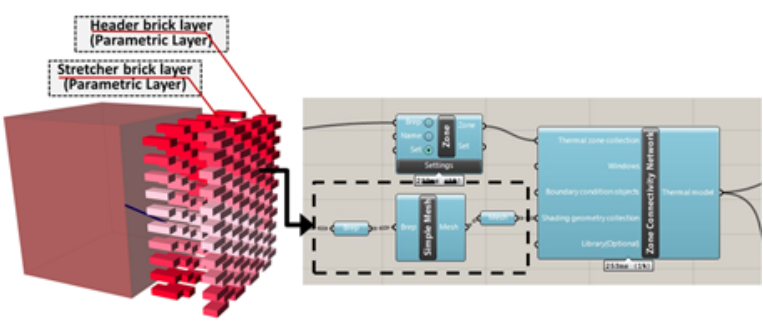

Figure 7: 3D model of cases resulting from attractor line connected to "Shading geometry collection" within ArchSim.

\section{Robotic Assembly Simulation}

The second section involves the robotic assembly simulation of the brick wall configuration. We use Grasshopper and KUKA's parametric robot control (PRC) component for Grasshopper to simulate the assembly process (Figure 8). After the brick wall parametric configuration is defined in the thermal simulation process, the Grasshopper script performs the following operations as a general assembly workflow:

First, based on the attractor curve logic of the nonuniform gradient distribution of brick wall configuration, the script generates the geometry for all custom bricks. A sorting algorithm then initiates the brick-laying automation process, where bricks are classified and stacked vertically on the ground floor. The definition of coordinates for allocating the stacks depends on the specific KUKA robot model (KUKA, 2014). In this paper, we used the KR 90 R2700 Pro model, with 6 controlled axes, a high payload (80 - 300 $\mathrm{kg}$ ), maximum reach of $2696 \mathrm{~mm}$, and custom tool length of $185 \mathrm{~mm}$. The robot was elevated on a $200 \mathrm{~mm}$ reinforced concrete base.

Since the brick configurations are all non-modular or unique due to the parametrically defined wall configuration (20 unique brick groups were used), the sequence and precision of brick allocation and placement is critical to correct assembly. 

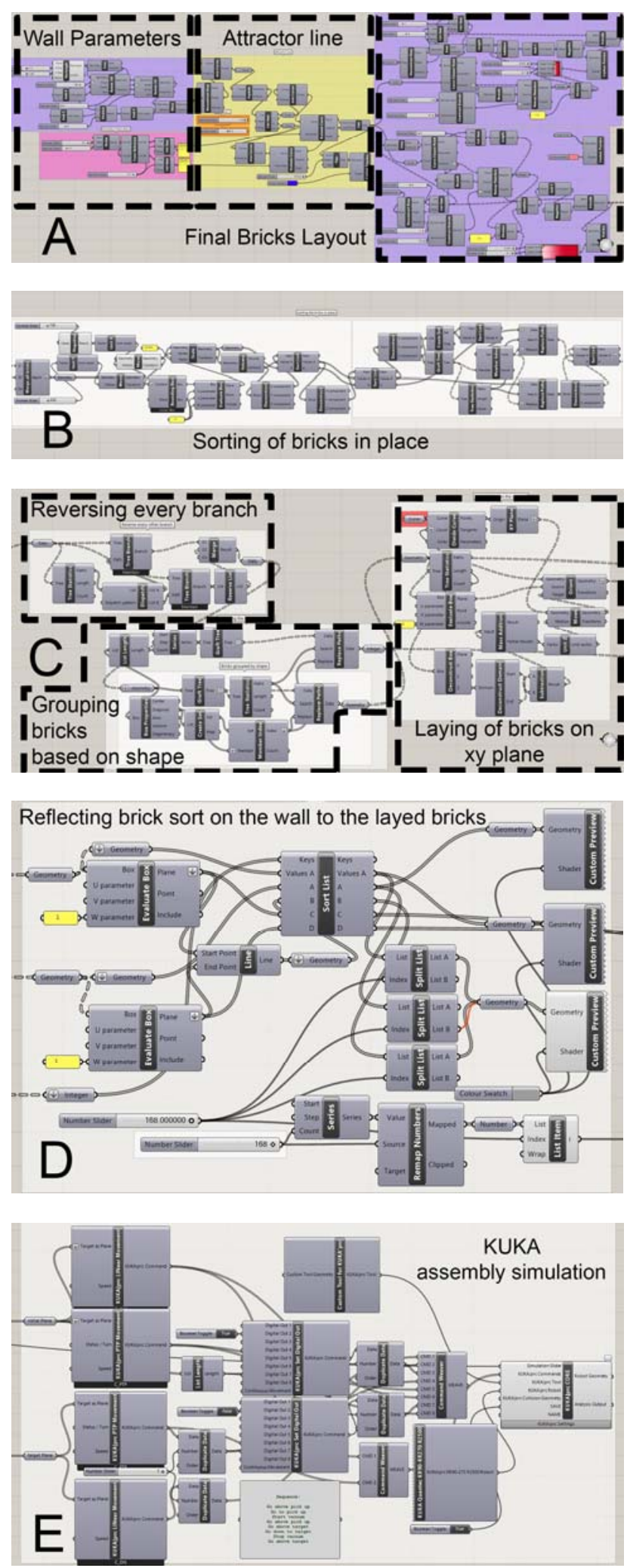

Figure 8: Grasshopper script for robotic assembly simulation (A: Wall design and simulation automation, B: Brick sorting, C: Grouping/laying bricks on ground floor plane, D: Mapping sorted bricks to vertical wall, E: KUKA assembly simulation).

An algorithm was thus developed to sort brick in place, identify groups of bricks in terms of sequence of assembly, group bricks by shape, lay bricks on ground floor plane, map all sorted bricks to the $3 \mathrm{D}$ coordinates of the vertical wall configuration, then perform assembly simulation. The KUKA parametric robot control (PRC) plugin (Braumann \& Brell-Cokcan, 2015) was used to simulate the assembly process, where bricks are picked and placed in their 3D wall location based on the parametric configuration logic. Figure 9 shows an extract of the robotic assembly script using KUKA PRC.

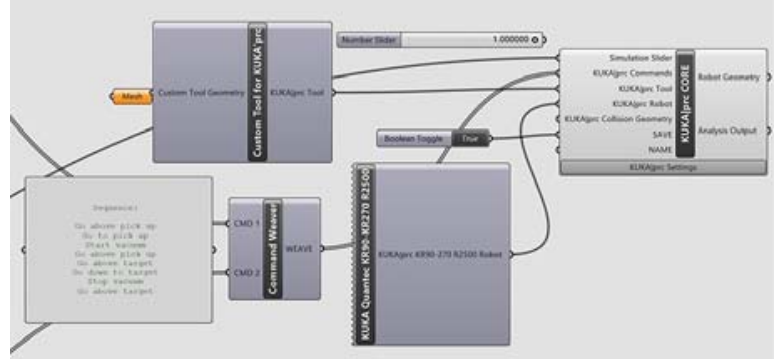

Figure 9: Extract of Grasshopper script for robotic assembly simulation using KUKA parametric robot control (PRC) component.

\section{Solar Radiation Simulation}

The base case model is a Flemish bond wall without any extruded bricks. The thermal performance of the base case is $131 \mathrm{kWh} / \mathrm{m}^{2}$. Based on previous work that focuses on studying thermal performance effect only of brick extrusion along the attractor curve, the thermal optimal case was found to be $27.75 \%$ better than the base case (Figure 10). The optimal and worst thermal performance cases are shown in Figure 11 (Tarabieh et al., 2018).

In this paper, solar radiation exposure was addressed by measuring the solar radiation in the center of each brick in the wall, resulting in 168 lighting sensors. The simulation was conducted for a whole year and the average results were presented. Three simulation phases are conducted to study the effect of wall topography and texture on both solar radiation of facade and thermal performance. Thermal mass, selfshading and solar radiation directly affect thermal performance. Thus, in this paper, solar radiation with thermal mass in wall topography are studied as an approach to enhance thermal performance in hot arid climates.

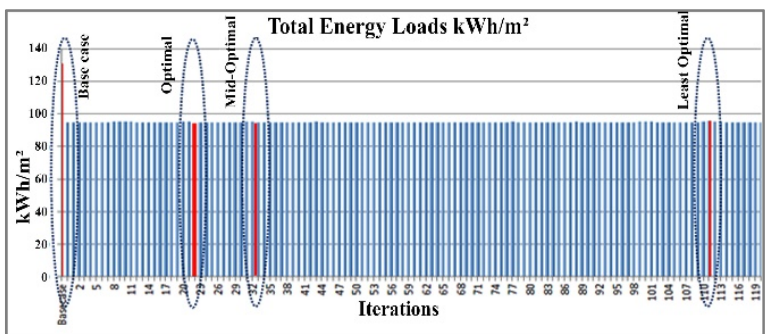

Figure 10: Comparing thermal performance of all tested cases to base case. 


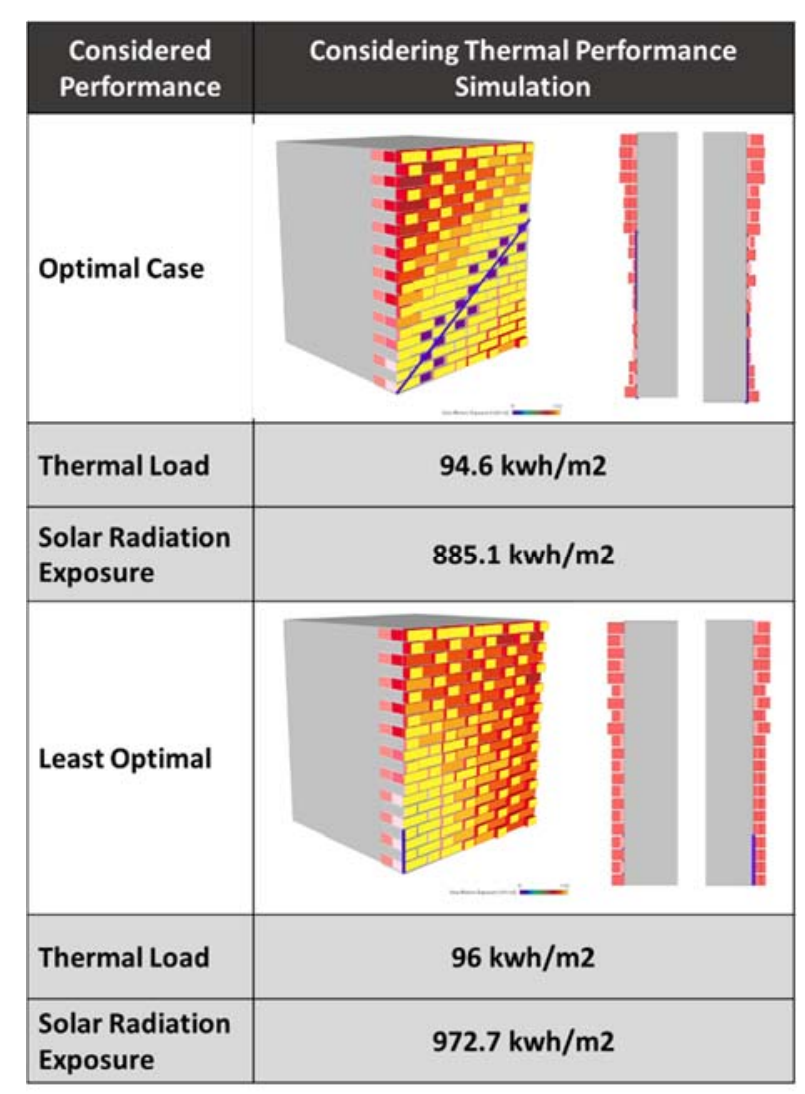

Figure 11: Optimal and least optimal thermal performance cases.

\section{Phase I}

In this phase, the solar radiation simulation was conducted for the 121 cases of the attractor curve. The best case of thermal performance at this stage was not the same best case of solar radiation. Parameters of wall topography used in the 121 cases to run solar radiation are shown in Table 2.

Table 2: Parameters of optimal case with respect to thermal performance.

\begin{tabular}{|c|l|}
\hline Parameters & Values \\
\hline Header brick & $0-10 \mathrm{~cm}$ with $1 \mathrm{~cm}$ increments \\
\hline Stretcher brick & $0-5 \mathrm{~cm}$ with $0.5 \mathrm{~cm}$ increments \\
\hline
\end{tabular}

By comparing the two best cases of each of the previous results in the thermal and the current solar radiation simulation, a negligible difference around $0.1 \mathrm{KWh} / \mathrm{m}^{2}$ in thermal performance was identified, but the difference in solar radiation between the two cases was $10.3 \mathrm{KWh} / \mathrm{m}^{2}$ (Figure 12).

The paper will therefore focus on studying this optimal case in more variations for wall topography in the next steps, as shown in Figure 13.

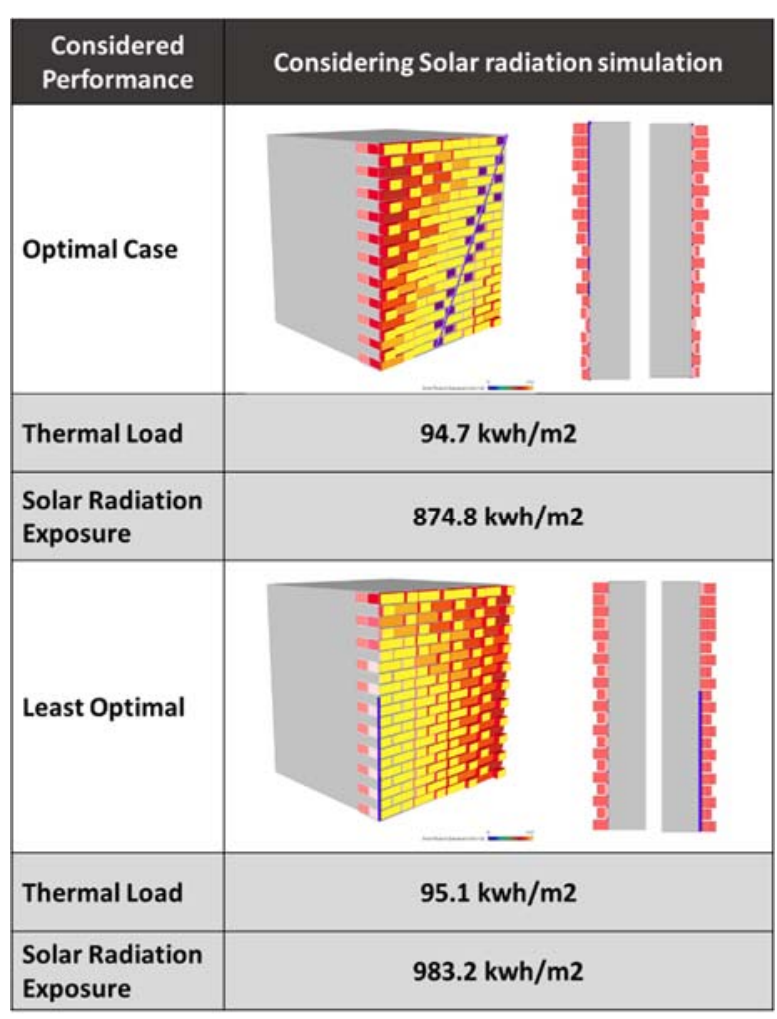

Figure 12: Parameters of optimal and least optimal cases with respect to solar radiation.

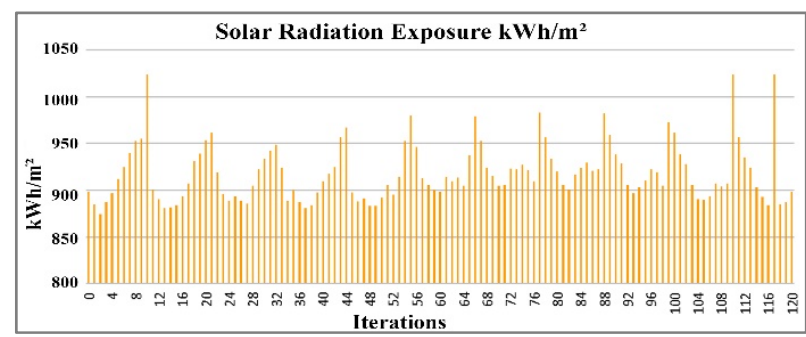

Figure 13: Comparison of solar radiation performance for all tested cases.

\section{Phase II}

Solar radiation and thermal performance in this phase target rougher wall topography with $2.5 \mathrm{~cm}$ extrusion increments on 5 steps. This gives space for more selfshaded surface area of the room. Wall parameters are shown in Table 3.

Table 3: Wall topography parameters for phase II.

\begin{tabular}{|c|c|}
\hline Parameters & Values \\
\hline Header brick & $\begin{array}{l}0-10 \mathrm{~cm} \text { with } 2.5 \mathrm{~cm} \text { increments } \\
(0,2.5,5,7.5,10)\end{array}$ \\
\hline $\begin{array}{l}\text { Stretcher } \\
\text { brick }\end{array}$ & $\begin{array}{l}0-5 \mathrm{~cm} \text { with } 2.5 \mathrm{~cm} \text { increments } \\
(0,2.5,5) \mathrm{cm}\end{array}$ \\
\hline
\end{tabular}


The wall topography and the results of thermal performance and solar radiation are shown in Figure 14.

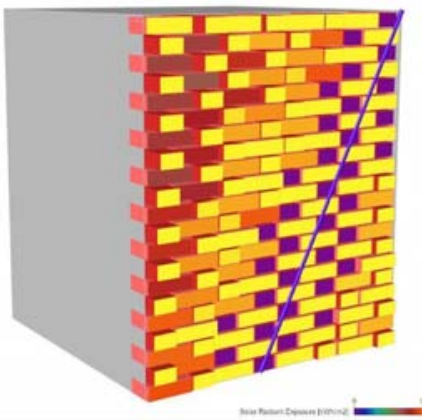

\section{Thermal Load}

Solar Radiation

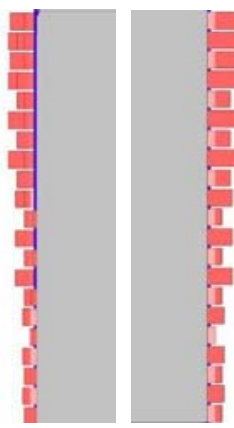

$94.5 \mathrm{kWh} / \mathrm{m}^{2}$

$771.5 \mathrm{kWh} / \mathrm{m}^{2}$

Figure 14: Results for phase II with respect to thermal and solar radiation.

\section{Phase III}

This phase targets the roughest topography in the tested cases. Two alternatives are studied; case A and B, where variations in extrusion values are between the header and stretcher.

\section{Case A}

The wall topography in this case tends to fix the header brick flat without any extrusion values, while the extrusion of stretcher bricks is done in five steps with $2.5 \mathrm{~cm}$ increments as in Phase II in relation to the attractor curve (Table 4).

Table 4: Parameters of case $3 A$.

\begin{tabular}{|l|l|}
\hline Parameters & Protrusion Values \\
\hline Header brick & 0 \\
\hline Stretcher brick & $\begin{array}{l}0-5 \mathrm{~cm} \text { with } 2.5 \text { increments } \\
(0,2.5,5) \mathrm{cm}\end{array}$ \\
\hline
\end{tabular}

The effect of solar radiation and thermal performance of this configuration is shown in Figure 15.

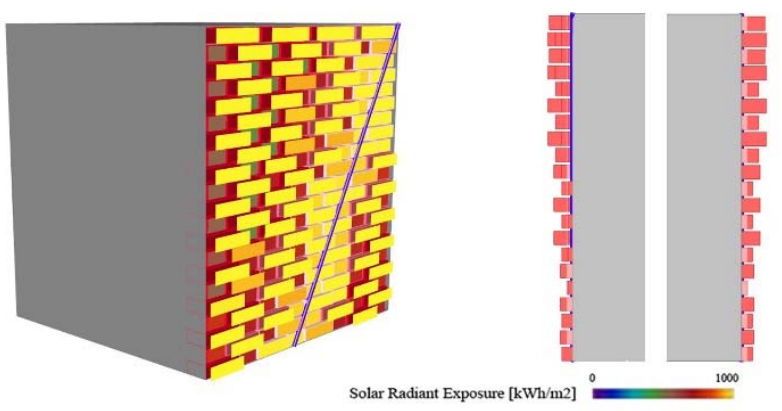

\begin{tabular}{|l|l|}
\hline Thermal Load & $93.7 \mathrm{kWh} / \mathrm{m}^{2}$ \\
\hline Solar Radiation & $586.9 \mathrm{kWh} / \mathrm{m}^{2}$ \\
\hline
\end{tabular}

Figure 15: Solar radiation and thermal performance results of Case $3 A$.

\section{Case B}

Case B tends to fix the stretcher bricks of the Flemish bond flat without extrusion as shown in Table 5, while having the extrusion of header bricks extruded on five steps with $2.5 \mathrm{~cm}$ increments as in Phase II in relation to the attractor curve, as shown in Figure 16.

Table 5: Parameters of Case 3B.

\begin{tabular}{|c|l|}
\hline Parameters & \multicolumn{1}{l|}{ Protrusion Values } \\
\hline Header brick & $\begin{array}{l}0-10 \mathrm{~cm} \text { with } 2.5 \text { increments } \\
(0,2.5,5,7.5,10)\end{array}$ \\
\hline Stretcher brick & 0 \\
\hline
\end{tabular}
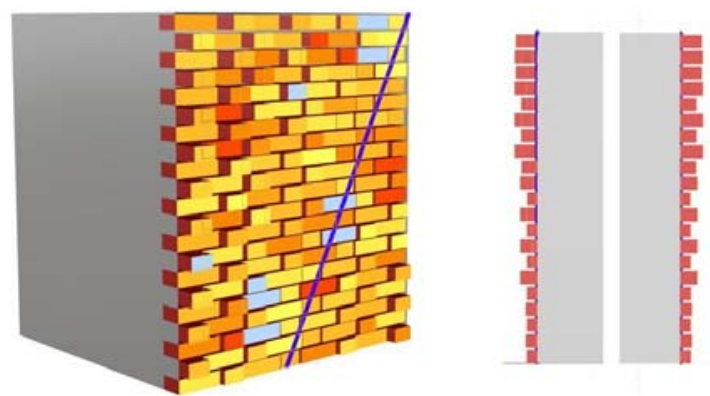

\begin{tabular}{|l|l|}
\hline Thermal Load & $94.4 \mathrm{kWh} / \mathrm{m}^{2}$ \\
\hline Solar Radiation & $585.2 \mathrm{kWh} / \mathrm{m}^{2}$ \\
\hline
\end{tabular}

Figure 16: Solar radiation and thermal performance results of Case $3 B$.

\section{Robotic Simulation}

Figure 17 shows the robotic assembly simulation process of the brick wall based on the thermally responsive wall configuration, demonstrated in sequence. First, the sorting process is initiated, and all bricks are grouped and laid on the ground floor plane. Bricks are then selected from unique brick groups based on their specific dimensions and the logic of the sequence of assembly. Finally, the bricks are assembled based on precision location in the $3 \mathrm{D}$ wall configuration. 


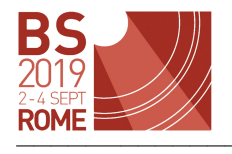

16th IBPSA
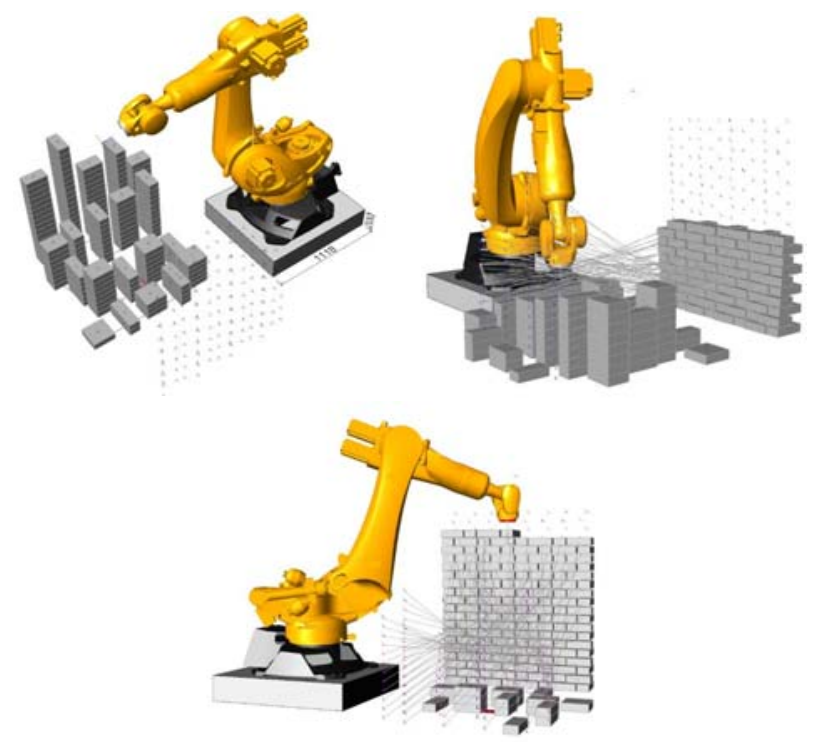

Figure 17: Robotic assembly simulation process of brick wall based on thermally responsive wall configuration (Top left: Sorting and grouping of bricks, Top right: Selection of bricks from unique brick groups, Bottom: Brick assembly based on precision location in wall configuration).

\section{Discussion of Simulation Results}

The simulation was conducted in three phases. The first phase applied solar radiation simulation for all the 121 cases resulting from the different attractor locations. The attractor line curve controlled the surface morphology resulting in different self-shading effects. Furthermore, the solar radiation results were analysed and compared to their thermal performance results from the previous study to select the balanced case that recorded a reduction on both thermal performance and solar radiation.

Although both sets do not select the exact surface morphology as the optimal case, but the configurations are quite similar. As a south oriented facade, the results if both sets showed that the optimal case is extruding bricks along the top edge, with some bricks extruded on the upper left corner that represents the western side. As shown in Figure 18 , the selected optimal case that could enhance both thermal performance and solar radiation exposure has a solar radiation exposure of $585.2 \mathrm{kWh} / \mathrm{m}^{2}$ compared to 1020 $\mathrm{kWh} / \mathrm{m}^{2}$ for a flat surface resulting in $43.4 \%$ enhancement.

This case also recorded a low energy consumption of 94.7 $\mathrm{kWh} / \mathrm{m}^{2}$ compared to the base case $131 \mathrm{kWh} / \mathrm{m}^{2}$ resulting in 27.7\% enhancement, as shown in Figure 19. The third phase (case A and case $\mathrm{B}$ ) have too close readings with a difference of $1.7 \mathrm{kWh} / \mathrm{m}^{2}$ in solar radiation and $0.7 \mathrm{kWh} / \mathrm{m}^{2}$ in thermal performance. The second and third phases aim at optimizing the solar radiation. It was found that the roughness of surface has a significant contribution on the solar gain rather smooth surfaces.

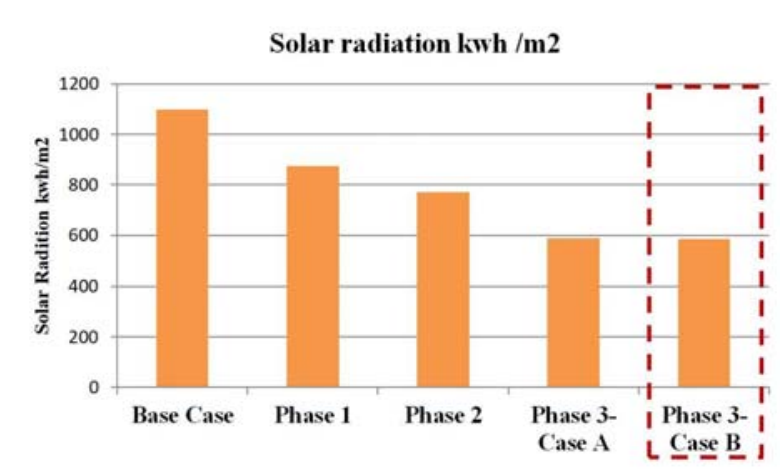

Figure 18: Comparing all tested cases of the three phases to the base case with respect to solar radiation.

Regarding the robotic simulation, the process proved effective regarding the desired precision of assembly, especially in a process that involves custom bricks and complex wall configuration and brick bonds. This was facilitated by the automation of numbering and sorting of the 20 unique brick groups which allowed for easy handling, construction and assembly during the robotic assembly process. This is especially significant, as all previous approaches have relied on the modularity of brick sizes and the systematic stacking and layering by the robotic assembly process, leaving gaps between the modular bricks. The challenge in this paper was to sort, number, pick, align and assemble custom bricks in a complex wall configuration.

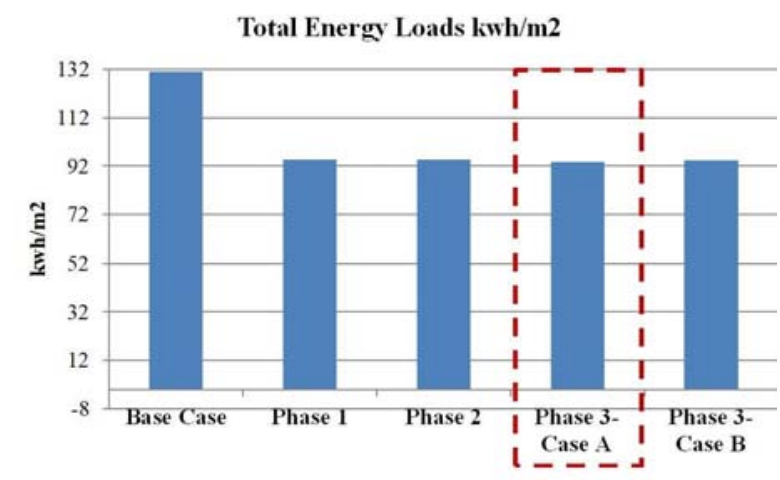

Figure 19: Comparing all tested cases of the three phases to the base case with respect to energy load.

The main limitations of the robotic simulation process in this case were related to the specifications of the robot, especially with respect to custom tool length and reach. The volume of stacked and sorted brick piles on the ground floor plane, which is proportional to the number of custom brick sizes, was significantly challenging in relation to the robotic simulation process in terms of smooth and efficient reach. Structural integrity of the assembled brick wall remains the most challenging for future research. 


\section{Conclusion}

Based on the coupling between parametric design and robotic simulation, we apply the developed methodology using a contextual case study to present a proof of concept and address the challenges of the simulation tools utilized, the output data generated, and the assembly process. The required coupling is achieved by means of a Grasshopper script that automatically translates the parametric brick wall variations resulting from thermal simulation using DIVA/ArchSim into the robotic assembly simulation process of the wall configuration. We used Grasshopper and KUKA's parametric robot control (PRC) component for Grasshopper to simulate the assembly process. The kinematic simulation revealed an effective assembly process with a high degree of precision in terms of coordinate allocation and desired brick wall configuration. Simulation allowed us to learn more about strategies for optimal configuration and proper KUKA models to perform actual building functions. Given the custom nature of the walls, challenges facing robotic assembly increase with span and reach requirements to handle bricks from different piles. This study is exploratory for working with both levels of complexity to prepare for proper coupling that can enhance capabilities of using simulation to predict problematic areas in assembly as well as optimal brick configuration. Through this research, several challenges emerged. The structural challenge was the major issue due to the custom nature of the assembled wall. Further testing needs to be applied especially with different wall configurations, depths, heights and brick bonds. Another challenge involves architectural aesthetics versus the robotic capability of assembly. This requires further testing to identify in depth the architectural limitations of the structural robotic assembly process.

\section{References}

Abdelwahab, S., \& Elghazi, Y. S. (2016) A generative performance-based design for low cost brickwork screens. Proceedings from BSO2016: Building Simulation and Optimization Conference. Newcastle (UK), 12-14 September 2016.

Braumann, J., \& Brell-Cokcan, S. (2015) Adaptive robot control: new parametric workflows directly from design to KUKA robots. Proceedings from eCAADe 33: Education and Research in Computer Aided Architectural Design in Europe. Vienna (Austria), 2015.

Cavieres, A., Gentry, R., \& Eastman, C. (2016) A processdriven representation schema for masonry wall assemblies. Proceedings from ISARC 2016: 33th International Symposium on Automation and Robotics in Construction, Auburn, AL (USA), 2016.

Dakhli, Z. and Z. Lafhaj (2017). Robotic mechanical design for brick-laying automation. Cogent Engineering 4(1): 1361600.
Dogan, T. (2013). ArchSim Energy Modeling for Grasshopper. https://es.aap.cornell.edu/ (Retrieved April 30, 2019).

Dörfler, K., Sandy, T., Giftthaler, M., Gramazio, F., Kohler, M., \& Buchli, J. (2016). Mobile Robotic Brickwork. In Reinhardt D., Saunders R., Burry J. (eds) Robotic Fabrication in Architecture, Art and Design 2016. Springer, Cham. Basel (Switzerland).

Fathy, F., Mansour, Y., Sabry, H., Abdelmohsen, S., \& Wagdy, A. (2015) Cellular automata for efficient daylighting performance: optimized façade treatment. Proceedings from BSO2015: Building Simulation and Optimization Conference. Hyderabad (India), 7-9 December 2015.

Gramazio, F., Kohler, M. \& Willmann, J. (2014). The Robotic Touch: How Robots Change Architecture. Park Books. Zurich (Switzerland).

Jakubiec, J., \& Reinhart, C. (2011). DIVA 2.0: Integrating daylight and thermal simulations using Rhinoceros 3D, Daysim and EnergyPlus. Proceedings from BS2011: International Building Performance Simulation Association Conference, Sydney (Australia), 14-16 November 2011.

KUKA, Robotics (2014), CODESYS Library for KUKA.PLC mx-Automation 2.0, KUKA.

McNeel, R. (2010). Grasshopper generative modeling for Rhino. http://www. grasshopper3d. com.

Sharif, S., Gentry, R., Eastman, C., \& Elder, J. (2015). Masonry unit database development for BIMmasonry. Proceedings from 12th North American Masonry Conference. Denver, CO (USA), 2015.

Sousa, J., Varela, P., and Martins, P. (2015). Between manual and robotic approaches to brick construction in architecture. Proceedings from eCAADe 33: Education and Research in Computer Aided Architectural Design in Europe. Vienna (Austria), 2015.

Tarabieh, K., Abdelmohsen, S., El-Ghazi, Y., ElDabaa, R., Hassan, A. \& Amer, M. (2017). Parametric investigation of three types of brick bonds for thermal performance in a hot arid climate zone. Proceedings from the Passive Low Energy Architecture Conference, Edinburgh (UK), 2017.

Tarabieh, K., Abdelmohsen, S., Hassan, A., El-Dabaa, R. \& El-Ghazi, Y. (2018). Parametric investigation of brick extrusion patterns using thermal simulation. Proceedings from BSO2018: Building Simulation and Optimization Conference. Cambridge (UK), 2018.

Usmanov., V., Bruzl, M., Svoboda, P. \& Šulc, R. (2017). Modeling of industrial robotic brick system. Proceedings from ISARC 2017: 34th International Symposium on Automation and Robotics in Construction, Taipei (Taiwan), 2017. 